\title{
Electron Microscopy Study of the Acid-Etched Titanium-Tissue Interface
}

\author{
K. Kubo*, W.-A. Chiou**, W. Att*, L.-C. Lai** and T. Ogawa* \\ *The Jane and Jerry Weintraub Center for Reconstructive Biotechnology, UCLA School of Dentistry, \\ Los Angeles, CA 90095 \\ **Dept. of Chemical Engineering and Materials Science, University of California, Irvine, CA 92697
}

Titanium implant therapy has been applied for several decades in oral/maxillofacial and orthopedic reconstruction. The quality of titanium-tissue interface is a crucial biological factor of bone-implant integration that directly affects the short- and long-term stability of endosseous implants. Although some ultrastructural approaches have been made to identify the structure of titanium-tissue interface [1], the interface with acid-etched surfaces, which are used most in current dental implant practice, has not been studied because of the critical, technical limitation of processing the tissue cross-sections. The purpose of this study is to characterize the microstructure at the interface between acid-etched titanium and cultured osteoblastic tissue using cross-section SEM and TEM techniques.

Grade 2 commercial pure titanium disks of $100 \mu \mathrm{m}$ thickness were acid-etched with sulfuric acid to a thickness of $50 \mu \mathrm{m}$. Bone marrow stromal stem cells were isolated from the femurs of 8-week-old male Sprague-Dawley rats and cultured on the thin titanium disk in the osteoblastic induction media. After culturing for 14 days, the samples were washed with 1xPBS and prepared for EM observation. The samples were trimmed and embedded in a BEEM capsule with Spurr embedding medium. Ultra-thin cross sections were microtomed with a diamond knife and collected on a thin carbon film to preserve the integrity of the ultra-thin sections. Ultramicrotomed thin sections were examined in a TEM while the thick sections were studied in a SEM.

SEM images show that the osteoblastic culture on the acid-etched titanium disk has a fibrous network with small globular structures (Figs.1a and 1b). Micro-scale surface roughness of titanium, with average peak-to-valley of $0.8 \mu \mathrm{m}$, was recognized in the cross-sectional images. The cross-sectional BSE (backscattered electron) image reveals a mineralized tissue layer on the surface of Ti. Both cross-sectional secondary electron and BSE images (Fig. 2) discriminated the electron contrast of the tissue from titanium, indicating that the biological tissue ranging $8-22 \mu \mathrm{m}$ in thickness was formed on the titanium surface. EDS line scan from the outer surface of tissue to the titanium interface revealed that elemental peaks of $\mathrm{Ca}$ and $\mathrm{P}$ existed in an identical level of vicinity (within 2 $\mu \mathrm{m}$ to the titanium surface), while the element of oxygen was distributed widely in the tissue with its peak at $3 \mu \mathrm{m}$ from the titanium surface. EDS elemental mapping confirmed a uniform Ca spread within the $2 \mu \mathrm{m}$ range of tissue. Cross-sectional TEM image (Fig. 3) shows an interfacial layer consists of two distinct tissue morphologies, platy (arrow) and needle-like (open arrows), developed at the Ti/tissue interface. Although the high magnification TEM image shows that there are poorly defined nanocrystallites mixed in the amorphous-like tissues on $\mathrm{Ti}$, the electron diffraction has a rather similar pattern of a typical hydroxylapatite (Fig. 4). Fibrous materials, presumably hydroxylapatite, were also seen at the tissue/Ti interface (Fig. 5). The TEM observation and electron diffraction indicate the onset of mineralization (crystallization) at the tissue/acid-etched Ti interface at 14 day of culture, and the fibrous needles may indicate the beginning stage of mineralization.

[1] Lei Saruwatari et al., J. Bone Miner. Res., 20, 2002-2016 (2005) 


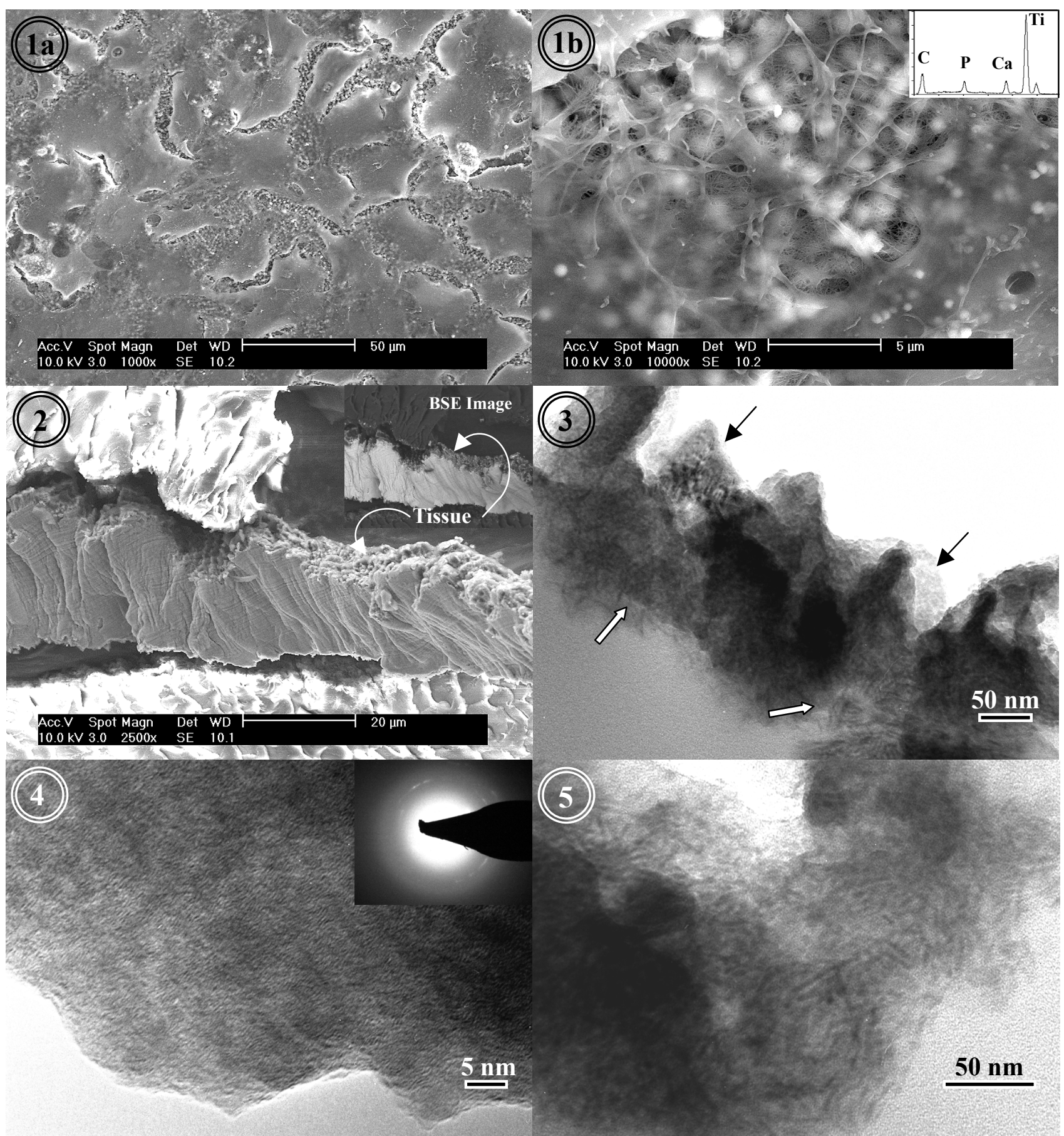

Fig. 1. Low (a) and high (b) mag. SEM images of cultured tissue on acid-etched Ti disk.

Fig. 2. Cross-sectional SEM images, SE and BSE, revealing a mineralized tissue layer on Ti disk.

Fig. 3. Low magnification TEM image showing tissues with different morphologies on Ti disk.

Fig. 4. High mag. TEM micrograph revealing poorly crystallized minerals in amorphous tissue.

Fig. 5. High mag. TEM image depicting fibrous needles with very faint Moire fringes. 